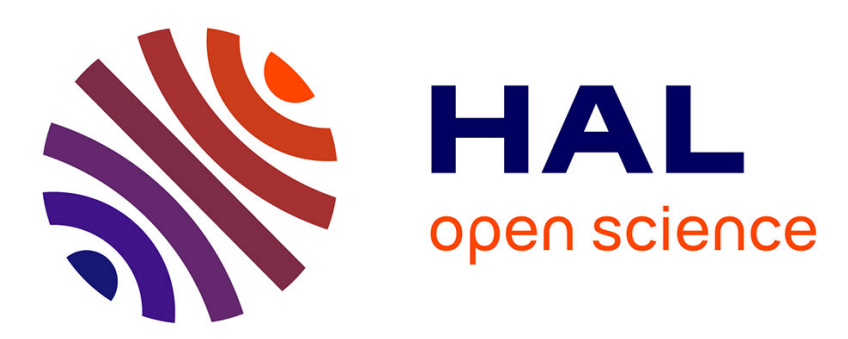

\title{
spalt-dependent switching between two cell fates that are induced by the Drosophila EGF receptor
}

\author{
Philip R Elstob, Véronique Brodu, Alex P Gould
}

\section{To cite this version:}

Philip R Elstob, Véronique Brodu, Alex P Gould. spalt-dependent switching between two cell fates that are induced by the Drosophila EGF receptor. Development (Cambridge, England), 2001. hal03031054

\section{HAL Id: hal-03031054 \\ https://hal.science/hal-03031054}

Submitted on 30 Nov 2020

HAL is a multi-disciplinary open access archive for the deposit and dissemination of scientific research documents, whether they are published or not. The documents may come from teaching and research institutions in France or abroad, or from public or private research centers.
L'archive ouverte pluridisciplinaire HAL, est destinée au dépôt et à la diffusion de documents scientifiques de niveau recherche, publiés ou non, émanant des établissements d'enseignement et de recherche français ou étrangers, des laboratoires publics ou privés. 


\title{
spalt-dependent switching between two cell fates that are induced by the Drosophila EGF receptor
}

\author{
Philip R. Elstob, Véronique Brodu and Alex P. Gould* \\ Medical Research Council, National Institute for Medical Research, Mill Hill, London, NW7 1AA, UK \\ *Author for correspondence (e-mail: agould@nimr.mrc.ac.uk) \\ Accepted 11 December 2000; published on WWW 7 February 2001
}

\section{SUMMARY}

Signaling from the EGF receptor (EGFR) can trigger the differentiation of a wide variety of cell types in many animal species. We have explored the mechanisms that generate this diversity using the Drosophila peripheral nervous system. In this context, Spitz (SPI) ligand can induce two alternative cell fates from the dorsolateral ectoderm: chordotonal sensory organs and non-neural oenocytes. We show that the overall number of both cell types that are induced is controlled by the degree of EGFR signaling. In addition, the spalt (sal) gene is identified as a critical component of the oenocyte/chordotonal fate switch. Genetic and expression analyses indicate that the SAL zincfinger protein promotes oenocyte formation and supresses chordotonal organ induction by acting both downstream and in parallel to the EGFR. To explain these findings, we

\section{INTRODUCTION}

One of the more surprising findings to come from the last twenty years of research is that only a relatively small number of highly conserved signaling molecules are used during animal development. This contrasts dramatically with the myriad of different cell types that are known to require induction by intercellular signals. Part of the explanation for this apparent discrepancy is that signaling pathways are multiply reused, each time producing a different outcome. Thus, one of the challenges for the future is to understand the context-dependent molecular mechanisms that link canonical signal transduction pathways to the induction of distinct cell fates.

Signaling via the epidermal growth factor receptor (EGFR) provides an impressive illustration of the wide range of cell types that can be induced by one highly conserved signaling pathway (reviewed by Perrimon and Perkins, 1997; Schweitzer and Shilo, 1997). For example, in the context of the developing Drosophila eye, the differentiation of at least seven distinct cell types is triggered by the reiterative use of the EGFR (Freeman, 1996). To account for at least part of this response diversity, it has been proposed that cell-type specificity is encoded in molecular differences that are generated prior to signaling (a prepattern; Dickson et al., 1992). Very recent work has identified the runt-domain transcription factor Lozenge as a propose a prime-and-respond model. Here, sal functions prior to signaling as a necessary but not sufficient component of the oenocyte prepattern that also serves to raise the apparent threshold for induction by SPI. Subsequently, sal-dependent SAL upregulation is triggered as part of the oenocyte-specific EGFR response. Thus, a combination of $\mathrm{SAL}$ in the responding nucleus and increased SPI ligand production sets the binary cell-fate switch in favour of oenocytes. Together, these studies help to explain how one generic signaling pathway can trigger the differentiation of two distinct cell types.

Key words: Oenocytes, Chordotonal organs, Drosophila melanogaster, EGF receptor, spitz (spi), spalt (sal)

component of such an EGFR prepattern (Flores et al., 2000; $\mathrm{Xu}$ et al., 2000). The emerging picture is that specific outputs from receptors such as the EGFR are generated in a combinatorial manner involving integration with other signaling inputs as well as transcription factor prepatterns (Halfon et al., 2000; Simon, 2000).

The EGFR also plays a critical role during the formation of a proprioceptive component of the Drosophila peripheral nervous system (PNS), the chordotonal organ. Indeed, the parallel between the formation of these sensory structures and photoreceptors extends way beyond the shared deployment of the EGFR. In both developmental contexts, the proneural gene atonal (ato) is expressed and required in the founding sensory cell (Jarman et al., 1993; Jarman et al., 1994). In the eye, the founder for each ommatidial cluster is the R8 photoreceptor whereas for the proprioceptors, the sensory mother cell is known as a primary chordotonal organ precursor (primary COP). ato-positive founder cells of both types produce SPITZ (SPI) ligand, a TGF $\alpha$-like molecule that activates the Drosophila EGF receptor (EGFR) in neighboring cells (Freeman, 1994; Tio et al., 1994; Okabe and Okano, 1997; zur Lage et al., 1997). This induction results in the recruitment of additional sensory cells: the secondary chordotonal organ precursors (secondary COPs), which contribute to proprioceptor arrays, and the majority of photoreceptor types in the eye ommatidium. In the case of chordotonal 
development, primary COPs express the transmembrane product of the rhomboid (rho) gene, which is required for the correct presentation of active SPI to the EGFR (Bang and Kintner, 2000). Recent results suggest that rhomboid family members also play a similar role in the eye (Wasserman et al., 2000). One important component of the response of recruited cells to the EGF signal is the production of the secreted EGFR inhibitor Argos (Golembo et al., 1996). Negative feedback by Argos spatially limits the activation of EGFR and thus provides a restriction on the number of cells recruited into each ommatidial or chordotonal cluster (Freeman, 1996; Okabe and Okano, 1997).

We have addressed the issue of how output specificity is achieved during EGFR signaling using a new model system, in which there is a choice between just two cell fates: the chordotonal organ and the oenocyte, a non-neural secretory cell. Genetic analysis indicates that, like secondary COPs, oenocytes require the functions of ato, rho and spi. We show that oenocyte precursors are induced as a single whorl of cells surrounding the most dorsal primary COP. In contrast, primary precursors that are more ventrally located recruit additional chordotonal precursors but not oenocytes. Manipulating EGFR signaling in a variety of ways can concomitantly increase or decrease the number of oenocytes and chordotonal organs but it is not sufficient to switch the qualitative nature of the EGFR response. In contrast, mutations in the spalt ( $\mathrm{sal}$ ) gene alter output specificity and lead to excess chordotonal recruitment at the expense of oenocyte formation. Epistasis tests and expression analysis reveal that sal acts both downstream and in a parallel pathway to EGFR signaling. To account for this, we present a prime-and-respond mechanism where the SAL protein is a critical component of an oenocyte nuclear prepattern and is also upregulated as part of the oenocytespecific EGFR response.

\section{MATERIALS AND METHODS}

\section{Fly stocks}

The following loss-of-function mutations were used: $\operatorname{argos}^{\Delta 7}$ (Freeman et al., 1992b), ato ${ }^{1}$ and Df(3R)p13 (Jarman et al., 1993; Jarman et al., 1994), spi ${ }^{l}$ (Nusslein-Volhard et al., 1984), rho ${ }^{P \Delta 5}$ (Freeman et al., 1992b) and a $r h o^{7 M 43}$ chromosome that also harbors a roughoid/rhomboid-3 mutation (Jurgens et al., 1984; Wasserman et al., 2000). The apparent null alleles $\mathrm{sal}^{445}$ and sal $^{16}$ (Jurgens, 1988; Kuhnlein et al., 1994) were used. Similar results were also obtained with $\mathrm{sal}^{l}$ (Nusslein-Volhard et al., 1984) except that the lateral chordotonal migration/orientation phenotype was not observed with this allele.

For misexpression, sal-GAL4 (Boube et al., 2000) and en-GAL4 (A. Brand, Wellcome/CRC Institute, Cambridge, UK) were used in combination with UAS-rho (de Celis et al., 1997; Wasserman et al., 2000), UAS-sspi (Schweitzer et al., 1995) and UAS-EGFR ${ }^{D N}$ (O'Keefe et al., 1997). Three independent insertions of UAS-sal (Kuhnlein and Schuh, 1996) were used (RS85, RS90 and RS91), each giving a qualitatively similar phenotype. $\mathrm{BO}$-lac $\mathrm{Z}$ is an oenocytespecific reporter line containing an enhancer from the sal complex (Barrio et al., 1999) and svp-lacZ refers to the $s v p^{d o n 1}$ enhancer trap (FlyBase, 1999). rho-lacZ refers to the X81 line (Freeman et al., $1992 b$ ), an enhancer trap insertion about 100 nucleotides from the rholacl insert that is already known to be $\mathrm{C} 1$ specific (Freeman et al., 1992a; zur Lage et al., 1997). pnt-lacZ refers to the pnt ${ }^{1277}$ enhancer trap into exon 1 of the pntP2 transcription unit (Scholz et al., 1993).

\section{Immunolabelling}

Embryo immunostaining was according to standard protocols using HRP or Alexa fluorescent conjugates (Molecular Probes). All fluorescent images were collected using confocal microscopy with a pinhole of 1. They are projections of several sections except Figs 1F, $2 \mathrm{~B}, 2 \mathrm{~F}$ and $3 \mathrm{~B}$ where single sections are shown. Primary antibodies used were: rabbit anti- $\beta$-galactosidase (Cappell) at 1:6000, mouse anti- $\beta$-galactosidase (Promega) at $1: 1000,22 \mathrm{C} 10 /$ anti-FUTSCH at 1:50 (Fujita et al., 1982; Hummel et al., 2000), anti-RHO (Sturtevant et al., 1996) at 1:600, anti-VVL (Anderson et al., 1995) at 1:1500, anti-SAL (Kuhnlein et al., 1994) at 1:30 or anti-SAL (Barrio et al., 1999) at 1:500.

\section{RESULTS}

The larval oenocytes of Drosophila are conspicuous secretory cells of ectodermal origin (Bodenstein, 1950; Hartenstein et al., 1992). They are arranged in clusters of, on average, 6 cells per abdominal hemisegment, occupying a characteristic lateral and subepidermal location. In contrast to the invariant peripheral nervous system, the number of cells in each larval oenocyte cluster can vary between 4 and 9 . Using many different molecular markers, we have traced the development of larval oenocytes (hereafter called oenocytes) from the third larval instar back to the extended germ band stage of embryogenesis (P. E. and A. G., unpublished). In brief, we have found that developing oenocytes express four genes from very early stages, all of which encode DNA-binding proteins. These are seven up (svp), pointed ( $p n t)$, spalt (sal) and ventral veins lacking $(v v l)$ which produce proteins of the nuclear receptor, ETS-domain, zinc-finger and POUhomeodomain class, respectively (Mlodzik et al., 1990; Scholz et al., 1993; Kuhnlein et al., 1994; Anderson et al., 1995).

\section{Oenocytes form around the most dorsal chordotonal precursor and require ato}

In the late embryo, immunolabelling experiments were carried out with two independent oenocyte markers: svp-lacZ, an enhancer trap into the svp gene and $B O$-lacZ, a regulatory construct containing an oenocyte-specific enhancer from the sal complex (see Materials and Methods). Using these markers, in conjunction with the sensory neuronal marker antiFUTSCH/22C10 (Fujita et al., 1982; Hummel et al., 2000), it can be seen that each cluster of oenocytes is closely associated with an array of five lateral chordotonal organs, termed an Lch5 (Figs 1A,B, 6A). In each abdominal hemisegment, there are eight chordotonal organs that are partitioned into arrays consisting of one dorsolateral ( $\left.\mathrm{V}^{\prime} \mathrm{ch} 1\right)$, five lateral (Lch5) and two ventral (VchAB) organs (Brewster and Bodmer, 1995). The close apposition of mature oenocyte clusters and Lch5 arrays in late embryos suggested that their formation might be linked in some way. In order to investigate this possibility, we examined the spatial relationship between the precursors of both cell types in early embryos.

Each chordotonal organ is formed by a single chordotonal organ precursor (COP) that divides asymmetrically to produce a sensory neuron, scolopale, ligament and cap cell (Fig. 6A, reviewed in Brewster and Bodmer, 1996). Previous studies using the rholacl reporter line (zur Lage et al., 1997) have determined that the progeny of the most dorsal COP (C1) 
constitute the most anterior chordotonal organ of the lateral cluster (Lch5a, Fig. 6A). We have used a similar rho-lacZ insertion that is also specific for Lch5a and its precursor COP (Fig. 1C-E and materials and methods), together with anti$\mathrm{SAL}$, to follow the development of $\mathrm{C} 1$ and oenocytes simultaneously. By stage 10, C1 has delaminated and does not express SAL, despite lying directly underneath a dorsal domain of SAL-positive ectoderm (termed the dorsal SAL domain, Figs 1D, 2B). By stage 11, C1 has already divided (zur Lage et al., 1997) and its progeny are surrounded by a whorl of sickle-shaped nuclei expressing higher levels of SAL than surrounding cells (Figs 1E,G, 2B). The whorl structure always appears in a dorsal and posterior segmental position, close to the ventral limit of the SAL domain, and corresponds to oenocyte precursors in the process of delamination (P. E. and A. G., unpublished). In addition to high levels of SAL, the oenocyte precursor whorl also expresses svp-lacZ and $v v l$ (Fig. $1 \mathrm{~F}$ and data not shown). As we only observe one oenocyte precursor whorl per hemisegment and this surrounds $\mathrm{C} 1$, we conclude that more ventral COPs (Okabe and Okano, 1997; zur Lage et al., 1997) are not associated with the formation of oenocytes (also see Discussion).

To test the idea that the $\mathrm{C} 1$ cell might have an influence on oenocyte development, ato mutants were examined. Embryos carrying ato $^{1}$, a strong hypomorph, in trans with a deficiency, have hemisegments in which the Lch5 array is either completely missing or reduced to a single lateral chordotonal organ, termed Lch0 and Lch1 phenotypes respectively (Jarman et al., 1995). Where an Lch1 is produced, this single organ is known to derive from a residual C1 precursor (zur Lage et al., 1997). We observe that oenocyte formation is completely abolished in those segments lacking all lateral chordotonal organs (Fig. 2A). Interestingly, in the majority of cases where an Lch1 is formed, it is associated with an oenocyte cluster of normal or somewhat reduced cell number. Similar results were obtained with ato ${ }^{1}$ homozygotes although the missing chordotonal/oenocyte phenotype is less penetrant (data not shown). Thus ato is required for oenocyte formation and there is a correlation between the presence of $\mathrm{C} 1$ and the ability to form an oenocyte cluster.

The above results do not rule out a scenario where ato is directly required in oenocyte precursors. However, given the correlation between the presence of $\mathrm{C} 1$ progeny and the formation of an oenocyte cluster in ato mutants, we think it more likely that ato functions in the $\mathrm{C} 1$ cell to facilitate oenocyte development in an indirect manner. This raises the possibility that an ato-dependent signal from $\mathrm{C} 1$ is involved in the induction of oenocyte precursors.

\section{Oenocyte induction requires rho, spi and EGFR activity}

$\mathrm{C} 1$ is one of a set of five primary COPs per hemisegment (C15, Fig. 6B) that delaminate from ato-positive proneural clusters. The continued expression of ato in these primary COPs switches on rho and thus activates SPI. In turn, this induces three secondary COPs via EGFR signaling, giving the full complement of eight COPs per hemisegment (Okabe and Okano, 1997; zur Lage et al., 1997). In rho mutants, where active SPI ligand is absent, the two lateral chordotonal organs that are descended from secondary COPs are missing, so producing an Lch3 phenotype (Bier et al., 1990). As C1 expresses rho and lies at the center of the whorl (Fig. 1E,F), oenocyte precursors are likely to be exposed to EGFR ligand. In addition, they express a reporter for pnt (Fig. 1G), a transcription factor target of the ras/MAP kinase pathway that is known to act downstream of the EGFR (O'Neill et al., 1994). For these reasons, we investigated the possibility that the EGFR pathway might be used to induce oenocytes.

Embryos homozygous for $r h o^{7 M 43}$ or $r h o^{P 45}$ display an Lch3 phenotype and a complete lack of oenocytes at stage 16 (Fig. $2 \mathrm{E}$ and data not shown). This is consistent with data presented in a previous analysis of rho function (see Fig. 3 from Wappner et al., 1997). Embryos lacking the EGFR ligand, Spi, also present an Lch3 phenotype (Rutledge et al., 1992) and again lack all oenocytes (Fig. 2F). The absence of mature oenocytes in spi and rho mutants can be traced back to stage 11 where the oenocyte precursor whorls are missing (Fig. 2B-D). Importantly, although SAL upregulation in oenocyte precursor cells is a response to EGFR signaling, the dorsal SAL domain remains in rho and spi mutants and therefore is independent of SPI ligand (Fig. 2C,D).

In order to test the role of the EGFR itself, we used en-GAL4 (Brand and Perrimon, 1993) to misexpress a dominant-negative form of this receptor that lacks the cytoplasmic kinase domain (EGFR $^{\mathrm{DN}}$, Freeman, 1996; O'Keefe et al., 1997). The en-GAL4 driver expresses in a domain containing all of the Lch5 and oenocyte precursors but not the founding COPs for V'ch1 or VchAB (P. E. and A. G., unpublished). Like rho and spi loss of function, misexpression of $\mathrm{EGFR}^{\mathrm{DN}}$ at both $25^{\circ} \mathrm{C}$ and $29^{\circ} \mathrm{C}$ resulted in a complete loss of oenocytes (Fig. 2G). In contrast, the lateral chordotonal organ array was only reduced by one at $25^{\circ} \mathrm{C}$ with the rho/spi Lch3 phenotype appearing only at the higher temperature (Fig. 2G). Thus, a reduction in EGFR activity can completely suppress oenocyte formation but only partially blocks secondary COP induction. This unexpected result suggests that the threshold for EGFR activation required to induce oenocytes may be higher than that for chordotonal precursors.

To test whether the EGFR is required directly in oenocyte precursors or more indirectly in $\mathrm{C} 1$, a sal-GAL4 driver was used (Boube et al., 2000). In contrast to en-GAL4, this particular driver line directs dorsal expression in a subset of oenocyte precursors but not in $\mathrm{C} 1$ (data not shown). Expressing the EGFR ${ }^{\mathrm{DN}}$ using sal-GAL4 at $29^{\circ} \mathrm{C}$ had no effect on chordotonal organ number but did produce a significant reduction in the number of oenocytes per cluster $(2.73 \pm 1.14$ (mean \pm s.d.) $n=25)$ relative to controls from the same experiment $(5.76 \pm 0.83 n=21)$. Thus, oenocyte precursors autonomously require EGFR function. This is consistent with the previous data indicating that they express a reporter for $p n t$, an EGFR and MAPK target gene that is essential for oenocyte production (Fig. 1G and data not shown).

These studies show that EGF pathway components are required for the formation of oenocytes, in addition to their previously characterized role in chordotonal organ recruitment. Together with the preceding results, we conclude that SPI ligand, secreted from $\mathrm{C} 1$, is required to activate the EGFR in the neighbouring ectoderm, in turn leading to oenocyte induction.

\section{Excess EGFR signaling increases the number of oenocytes and chordotonal organs}

Why does an oenocyte whorl form around $\mathrm{C} 1$ and not around more ventrally located primary COPs? Previous studies have 


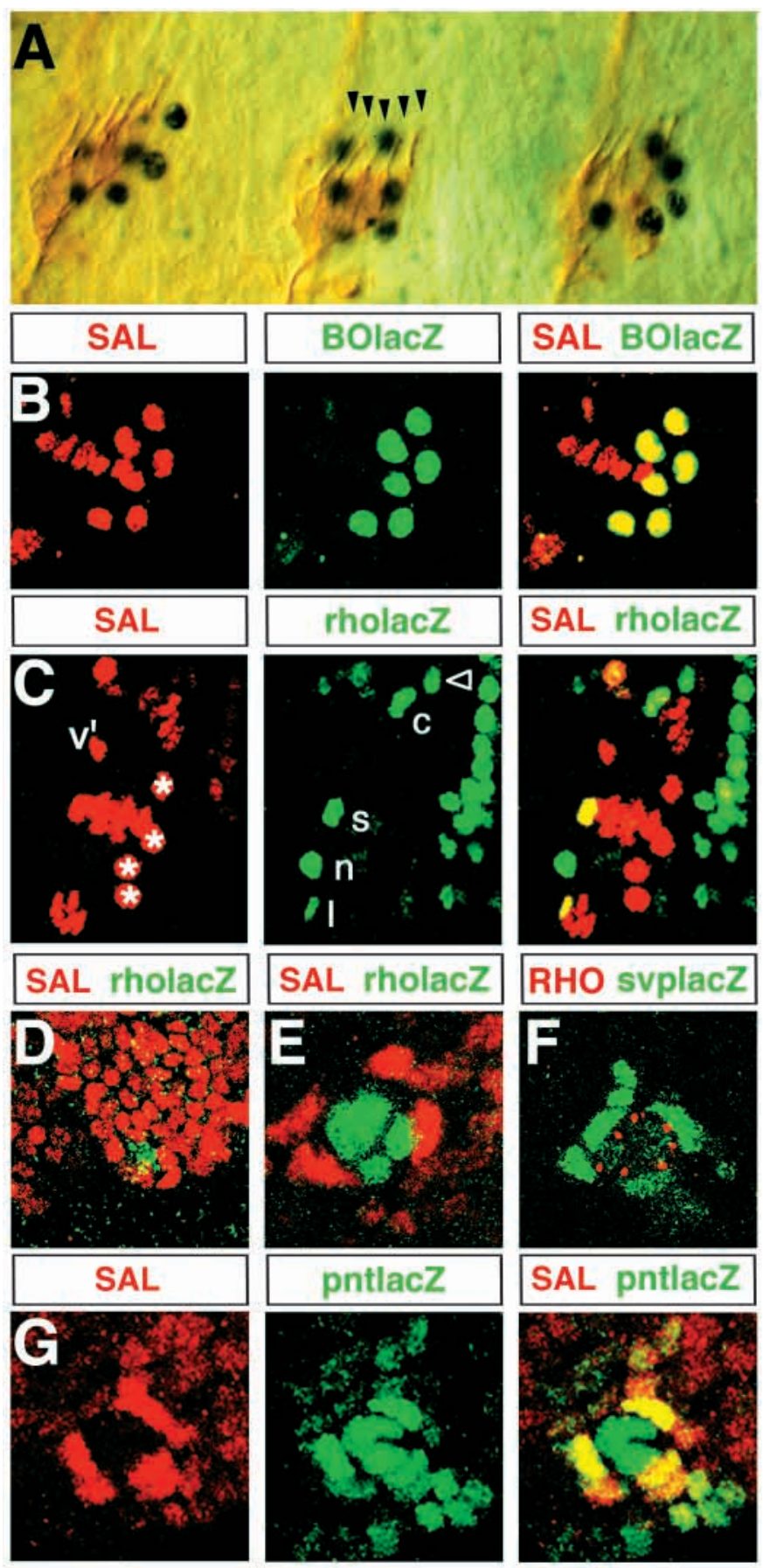

shown that rho and thus active SPI ligand is expressed in C2C5 (Okabe and Okano, 1997; zur Lage et al., 1997) yet these cells only recruit chordotonal organs and not oenocytes. In light of the EGFR ${ }^{\mathrm{DN}}$ results, a greater degree of signaling is likely to be required to trigger oenocyte induction than secondary COP recruitment. Consistent with this, $\mathrm{C} 1$ is known to express rho more strongly than other COPs (zur Lage et al., 1997) and this could provide the trigger for the induction of oenocytes rather than COPs. To test this possibility, we examined argos mutants where the degree of EGFR signaling should be increased in the vicinity of all primary COPs. Removing argos function resulted in one extra lateral
Fig. 1. Oenocytes are associated with the Lch5 array and form around the $\mathrm{C} 1$ primary precursor. Anterior is to the left and dorsal is to the top in this and all subsequent figures. (A) Three abdominal segments of a stage 16 embryo showing clusters of oenocytes expressing svp-lacZ (black) that are closely associated with lateral chordotonal organs. Lch5 neurons are labeled with the neuronal marker anti-FUTSCH/22C10 (brown). Arrowheads indicate the five dendrites of the Lch5 neurons (see Fig. 6A). (B) SAL labels oenocytes and non-neuronal cells of the Lch5 at stage 16. Anti-SAL overlaps with $\mathrm{BO}$-lacZ (see Materials and Methods) in large oenocyte nuclei but SAL also uniquely labels the smaller, more internally located, nuclei of the Lch5 non-neuronal cells. (C) rholacZ labels the most anterior lateral chordotonal organ, Lch5a. The ligament (1), scolopale (s), and cap cell (c) express both SAL and rho-lacZ. In addition, rho-lacZ labels the Lch5a neuron (n) and one extra cell (open arrowhead) that probably corresponds to an attachment cell (Brewster and Bodmer, 1995). Note that rho-lacZ expression does not overlap with SAL expression in the Lch5b-e, the $\mathrm{V}^{\prime}$ ch1 ( $\mathrm{V}^{\prime}$, indicates scolopale cell), or the oenocytes (asterisks). (D-F) $\mathrm{C} 1$ daughter cells that express RHO lie at the center of the oenocyte precursor whorl. (D) Weak rho-lacZ expression is first observed in the $\mathrm{C} 1$ precursor at late stage 10 underlying a domain of SAL expression in the dorsal ectoderm. (E) During stage 11, C1 divides and SAL upregulation is now seen in large sickle-shaped nuclei surrounding three $\mathrm{C} 1$ daughter cells. (F) Nuclei of the oenocyte precursor whorl, marked with svp-lacZ, surround cells expressing RHO that is localized in membrane plaques.

(G) Oenocyte precursors and $\mathrm{C} 1$ daughter cells express the MAPK target, pnt-lacZ. Anti-SAL overlaps with pnt-lacZ expression in the oenocyte precursor whorl at stage 11 . The progeny of $\mathrm{C} 1$ at the centre of the whorl also express pnt-lacZ but not SAL.

chordotonal organ (Fig. 3C) as previously described (Okabe et al., 1996). In addition, we observed that in argos mutants, a single oenocyte precursor whorl is present but it is expanded in diameter from one to two concentric rings of cells and thus gives rise to a large oenocyte cluster of 15-27 cells (Fig. 3A,B). Hence, removal of an EGFR inhibitor leads to an increase in oenocyte number but not to the induction of ectopic oenocyte whorls.

A second way of increasing EGFR activation is to misexpress rho using en-GAL4. As rho is rate limiting for spi activation, this should produce high levels of EGFR ligand in ectodermal cells throughout the dorsoventral axis. An Lch6 phenotype and an enlarged whorl producing a cluster of 17-27 oenocytes were observed (Fig. 3D-F) as in argos mutants. A more direct way of generating constitutively active EGFR ligand is to use a secreted form of the spi gene, sspi (Schweitzer et al., 1995). Driving sspi using en-GAL4 results in EGFR activation in the responding ectoderm that is COP independent. As with rho misexpression, an enlarged oenocyte precursor whorl results, giving rise to a giant cluster of 21-39 oenocytes (Fig. 3G,H). In these embryos, the PNS is highly disorganized but it is possible to ascertain that excess lateral chordotonal organs are formed, frequently giving an Lch6-7 phenotype (Fig. 3I). Interestingly, in UAS-sspi but not argos embryos, sal is upregulated dorsal to the whorl of sickle-shaped nuclei. However, neither sal upregulation nor the production of oenocyte precursors is ever observed outside the dorsal SAL domain. Thus, while excess EGFR ligand can dramatically increase the number of oenocytes that are recruited around $\mathrm{C} 1$, it is not sufficient to induce ectopic oenocyte precursors around other primary COPs. We surmise that factor(s) other than SPI 


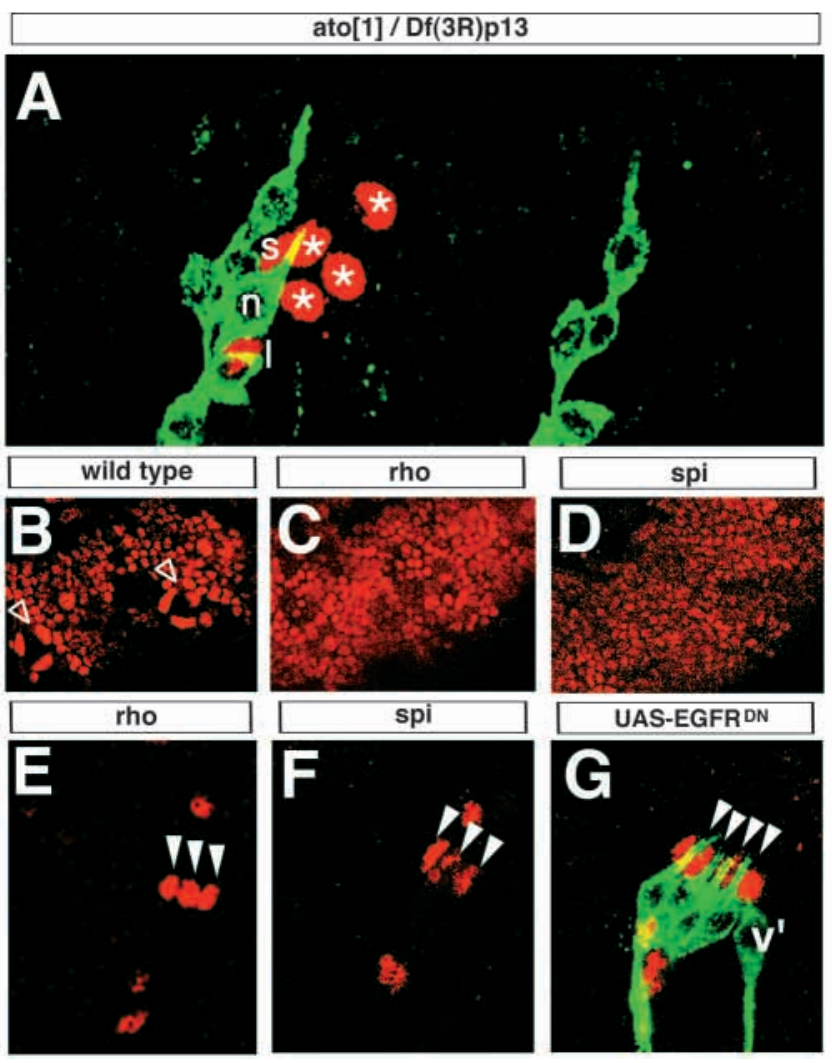

ligand must limit the oenocyte response to the dorsal SAL domain.

\section{sal promotes oenocyte induction and limits the number of lateral chordotonal organs}

Given that manipulating EGFR activation does not switch the choice of recruited cell from COP to oenocyte, there could be a dorsoventral difference in the competence of the ectoderm to respond to EGFR ligand. As oenocytes can only be induced within SAL territory, even in the presence of excess EGFR signaling, it could be that sal itself is involved in the dorsal restriction process. To test this, we examined embryos carrying the null alleles $\mathrm{sal}^{16}$ and $\mathrm{sal}^{445}$ (Kuhnlein et al., 1994). Using both svp-lacZ and anti-VVL markers, almost no oenocytes were seen (Fig. 4A,B and data not shown) and with svp-lac $Z$ we observed that this deficit is manifest as early as the whorl stage (data not shown). This provides a clear demonstration that oenocyte induction

Fig. 3. Loss of argos or excess rho or spi lead to recruitment of supernumerary oenocytes and chordotonal organs. (A-C) In argos mutants at stage 11, the precursor whorl is enlarged and contains many extra cells (A) and by stage 16 oenocyte clusters containing $15-27$ cells $(20 \pm 4.2, n=10)$ are present (B). Loss of argos function also results in one extra lateral chordotonal organ, giving an Lch6 phenotype (C). (D-I) Overexpression of UAS-rho (D-F) or UAS-sspi (G-I) using en-GAL4 produces an enlarged whorl at stage $11(\mathrm{D}, \mathrm{G})$ similar to those seen in argos mutants. By stage 16, giant clusters of $17-27(23 \pm 2.7, n=9 ; \mathrm{E})$ or $21-39(29 \pm 4.6, n=21 ; \mathrm{H})$ oenocytes are observed that are associated with disorganized Lch6-7 arrays (F,I). UAS-sspi produces SAL upregulation throughout the dorsal sal domain but sickle-shaped nuclei are restricted to the normal whorl position $(G)$. Asterisk indicates a sensory neuron of uncertain identity.
Fig. 2. Oenocyte formation requires the functions of ato, rho, spi and the EGFR. In all panels SAL and FUTSCH/22C10 expression are shown in red and green, respectively. White arrowheads indicate the position of each lateral chordotonal organ in this and subsequent figures. (A) ato $1 / \mathrm{Df}(3 \mathrm{R}) \mathrm{p} 13$ embryo at stage 16 . The anterior segment contains four oenocytes (asterisks) together with a single lateral chordotonal organ with the neuron (n) expressing FUTSCH/22C10 and the ligament (1) and scolopale (s) cells expressing SAL. The posterior segment contains no oenocytes and no lateral chordotonal organs, although other FUTSCH-expressing sensory neurons do remain. This represents the phenotype displayed in approximately $25 \%$ of hemisegments. The mixture of Lch0 and Lch1 seen at stage 16 in ato $1 / \mathrm{Df}(3 \mathrm{R}) \mathrm{p} 13$ correlates with the stage 11 phenotype where some segments contain oenocyte precursor whorls but others contain no recognizable whorl (data not shown). (B-D) At stage 11, oenocyte precursor whorls of large sickle-shaped nuclei expressing high levels of SAL are seen in wild type (B) but not in $r h o^{7 M 43}$ (C) or spi (D) homozygotes. However, the dorsal domain of moderate SAL expression is still present in these two mutants. Within this domain, some rings of nuclei expressing moderate but not high levels of SAL are observed but these lie in the wrong anteroposterior position to correspond to oenocyte precursors. Open arrowheads indicate whorls. (E,F) At stage 16, rho ${ }^{P \Delta 5}(\mathrm{E})$ and spi $(\mathrm{F})$ embryos display a fully penetrant Lch3 phenotype and lack all oenocytes. (G) A stage 15 embryo carrying UAS- EGFR ${ }^{D N}$ and enGAL4 at $25^{\circ} \mathrm{C}$. At this temperature, a partial block in EGFR activity is observed, producing an Lch4 phenotype. In addition, no oenocytes are formed.

requires $s a l$ and also reveals that svp lies downstream of sal in the oenocyte response cascade.

In addition to the lack of oenocytes, sal mutants display a highly penetrant Lch6-7 phenotype (Fig. 4C,D). In contrast to argos mutants, extra chordotonal organs are restricted to the lateral cluster with the other abdominal chordotonal organs $\left(\mathrm{V}^{\prime} \mathrm{ch} 1, \mathrm{~V}\right.$ ch $\left.\mathrm{AB}\right)$ remaining wild type in number (data not shown). This is consistent with the finding that the primary precursors for these more ventral chordotonal organs are $\mathrm{C} 4$
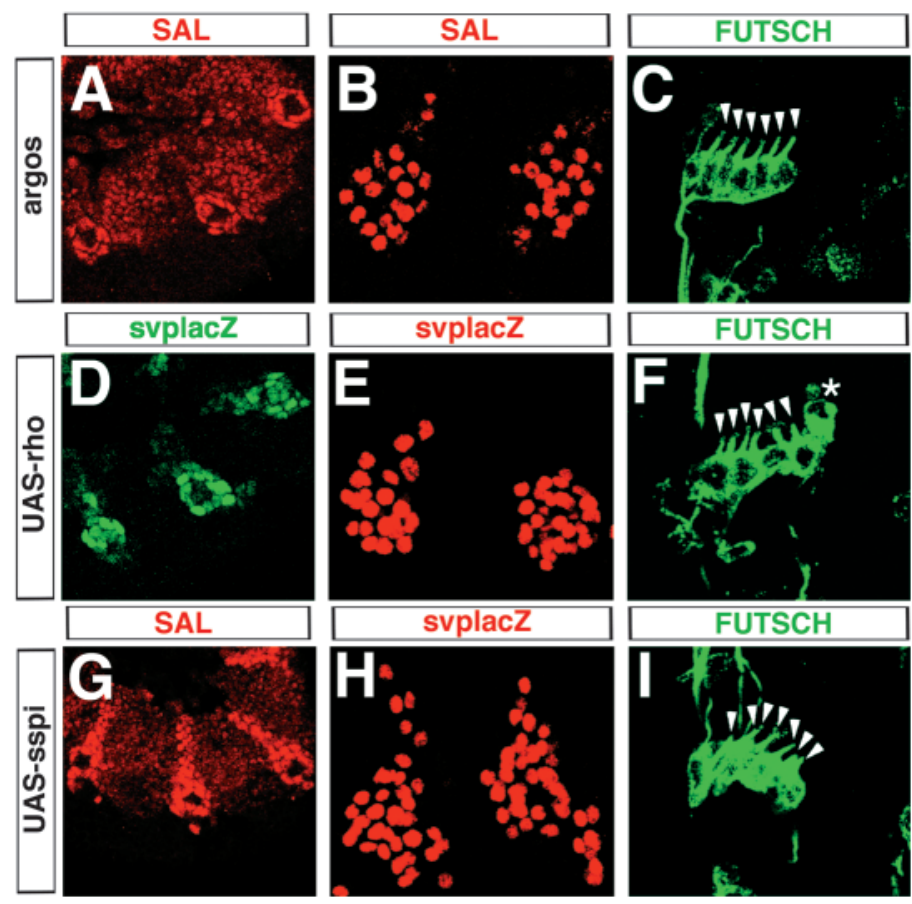


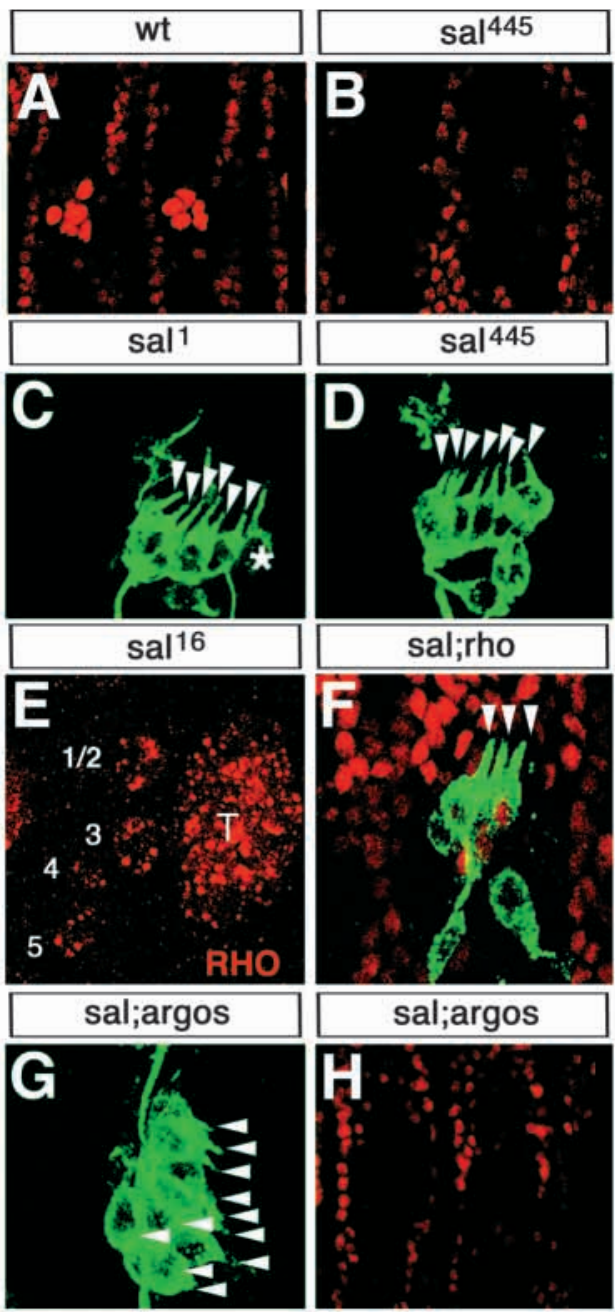

Fig. 4. sal acts downstream or in parallel to the EGFR to promote oenocyte and repress COP fates. The expression of VVL (red) marks oenocytes and FUTSCH/22C10 (green) labels chordotonal organs in all panels except E. (A,B) VVL expression indicates that, compared to wild type (A), $s a l^{445}$ mutants lack oenocytes (B). (C,D) Both $s a l^{1}$ (C) and $s a l^{445}$ (D) mutants display extra lateral chordotonal organs. Examples of an Lch6 and an Lch7 phenotype are shown. Asterisk indicates a sensory neuron of uncertain identity. (E) The expression of RHO in membrane plaques of the primary COPs (numbered) at late stage 10/early stage 11 appears normal in sal $^{16}$ mutants. $\mathrm{T}$ indicates the position of the tracheal placode/pit, a site of strong RHO expression. At later stages, high levels of RHO persist in C1 but not other COPs (data not shown). (F) In sal; rho double mutants, oenocytes are completely missing and a fully penetrant Lch3 phenotype is observed. $(\mathrm{G}, \mathrm{H})$ sal; argos double mutants display a dramatic increase in lateral chordotonal organs, an example of a disorganized Lch11 is shown $(\mathrm{G})$. In addition, these double mutants lack all oenocytes $(\mathrm{H})$.

and C5 (zur Lage et al., 1997), which both lie outside the dorsal SAL domain. In $s^{4} l^{445}$ mutants, we also observed that some of the lateral chordotonal organ arrays are displaced dorsally and frequently point ventrally (data not shown).

The phenotypic analysis above demonstrates that sal plays a positive role in oenocyte induction and a negative role in chordotonal organ formation. However, further studies are required to determine whether sal functions in the induced cells

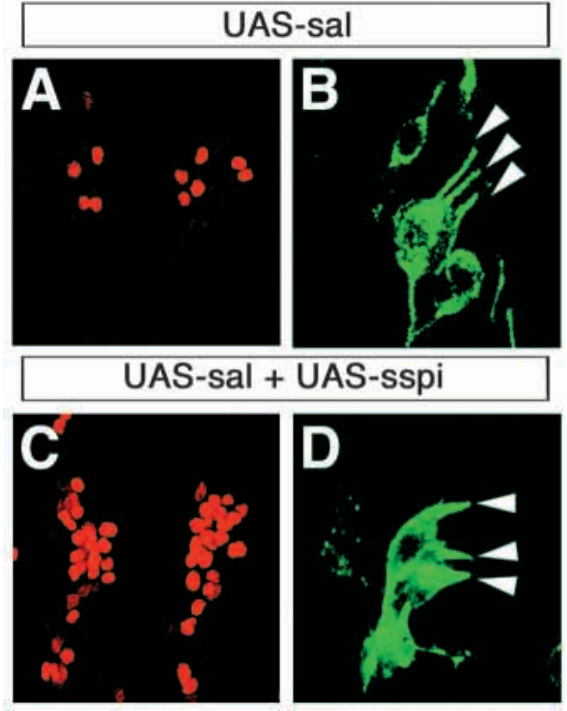

Fig. 5. sal is sufficient for COP repression but not oenocyte induction. Expression of svp-lacZ is shown in red and FUTSCH/22C10 is in green. (A,B) Misexpression of sal using en-GAL4 with the UAS-sal (RS90) insertion at $25^{\circ} \mathrm{C}$ produces a modest reduction in oenocyte number (A) with a mean of 4.6 cells per cluster $( \pm 1.2, n=25)$ and a mixed Lch3/4 phenotype (B). An example of a 6-cell and 4-cell oenocyte cluster is shown in A and an Lch3 in B. (C,D) Combined misexpression of $U A S$-sal (RS85) and UAS-sspi using en-GAL4 at $25^{\circ} \mathrm{C}$ results in giant oenocyte clusters of $21-35$ cells $(28 \pm 4.7, n=14)$. A highly penetrant Lch3 phenotype is also seen (D).

themselves or alternatively in the primary precursor producing the SPI signal.

\section{sal acts downstream or in parallel to the EGFR}

To investigate the regulatory relationship between the EGFR pathway and the functions of sal, the expression of RHO was examined in a sal ${ }^{16}$ background. The pattern and level of membrane-plaque associated RHO in sal mutant COPs appears indistinguishable from wild type (Fig. 4E and data not shown). As rho expression is rate limiting for active SPI production, this observation suggests that EGFR ligand synthesised in primary COPs is independent of sal. This is consistent with the observation that SAL is not detected in delaminated COPs at stage 11 (Fig. 1D,E and data not shown).

Addressing the question of whether the extra lateral chordotonal organs observed in sal mutants derive from primary or secondary COPs, we generated embryos mutant for both sal $^{445}$ and rho. These sal; rho double mutants lack all oenocytes and display an Lch3 phenotype that is identical to rho or spi single mutants (Fig. 4F). As the rho mutation abolishes secondary but not primary COP formation, the 1-2 extra lateral chordotonal organs formed in sal mutants must be derived from supernumerary secondary COPs. Furthermore, the sal; rho chordotonal phenotype indicates that rho loss of function is epistatic to sal loss of function. Together with the observation that RHO expression is normal in a sal background, we conclude that sal functions downstream or in a parallel pathway to the EGFR signal to repress chordotonal recruitment.

To explore further the relationship between sal and the EGF pathway, sal ${ }^{445}$; argos double mutants were produced. These 
have a dramatic chordotonal overproduction phenotype that is more severe than that seen in either single mutant. Frequently there are 9 or more lateral chordotonal organs per hemisegment that, as in sal mutants, are often scattered in lateral and dorsal positions (Fig. 4G and data not shown). This implies that the EGFR pathway is hyperactivated within the dorsal territory surrounding $\mathrm{C} 1$, where sal would normally repress secondary COPs and promote oenocytes. Importantly, the sal; argos genotype also abolishes oenocyte formation (Fig. 4H). This demonstrates that, for oenocyte induction, sal loss of function is epistatic to argos loss of function. Hence, despite the high levels of EGFR signaling around $\mathrm{C} 1$ that lead to excess secondary COP production, no oenocytes are recruited. These results strongly suggest that sal is required in the responding cell, either downstream or in parallel to the EGFR, for the induction of the oenocyte fate.

\section{SAL raises the apparent threshold for induction by SPI}

To test further the idea that sal biases the choice of EGFR recruited fate from COP to oenocyte, sal was misexpressed in the ectoderm overlying more ventral COPs using en-GAL4. At $25^{\circ} \mathrm{C}$, this results in a high frequency of Lch3-4 arrays (Fig. 5B) and at $29^{\circ} \mathrm{C}$ these become predominantly of the Lch3 type (data not shown). The Lch3 phenotype is identical to that seen with rho and spi and is therefore consistent with a loss of secondary COP recruitment. This is opposite

Fig. 6. Summary and prime-and-respond model for oenocyte and chordotonal induction. (A) Summary of the mean number of oenocytes (red) and lateral chordotonal organs (green) present in each abdominal hemisegment in the wild-type (WT) and mutant backgrounds indicated. In the wild-type panel the relative positions of the cap(c), scolopale (s), neuron (n) and ligament (1) cells that constitute each of the five organs (a-e) of the Lch5 are shown. Missing lateral chordotonal organs and oenocytes are indicated by unfilled outlines and the UAS results shown are with the en-GAL4 driver. (B) The prime-andrespond model. The central panel indicates the position of the five ATO-expressing primary COPs (C1-C5) relative to the dorsal oenocyte prepattern of low SAL expression. C2 is shown half obscured as it is not clear if it lies within or just ventral to the SAL domain. Either way, C2 does not express rho strongly (zur Lage et al., 1997) and therefore is unlikely to induce oenocytes. C1-3 contribute to the Lch 5 but $\mathrm{C} 4$ and $\mathrm{C} 5$ (dashed circles) do not. The left panel shows the induction of oenocytes $(\mathrm{O})$ via strong and persistent SPI signaling (large blue arrows) from $\mathrm{C} 1$ to the EGFR in overlying ectodermal cells. The oenocyte prepattern of low SAL raises the apparent threshold for induction by SPI. Low SAL also serves to prime the responding cell so that sal can be subsequently upregulated as part of the response to EGFR activation. In turn, this stimulates the expression of the sal target gene, svp (not shown). The right panel shows the induction of secondary COPs $\left(2^{\circ}\right)$ by moderate SPI signaling (small blue arrows) from $\mathrm{C} 3$ to ectodermal cells that are SALnegative. In this case, EGFR stimulation does not lead to the activation of sal or svp. Instead, sensory organ precursors that divide and differentiate into lateral chordotonal organs are produced.

$\mathrm{B}$ to the phenotype of sal loss-of-function mutants, and implies that during normal development sal plays a role in repressing COP recruitment around $\mathrm{C} 1$. In addition to the Lch3 phenotype, sal misexpression is also associated with a moderate reduction in oenocyte number (Fig. 5A). Examination of stage 11 embryos indicates that the oenocyte deficit can be traced back to the precursor whorl stage (data not shown). To confirm that this effect is not insertion-site specific, three different UAS-sal lines of varying strengths were tested at $25^{\circ} \mathrm{C}$ and gave a range of mean oenocyte cluster sizes that were all less than wild type (data not shown). One
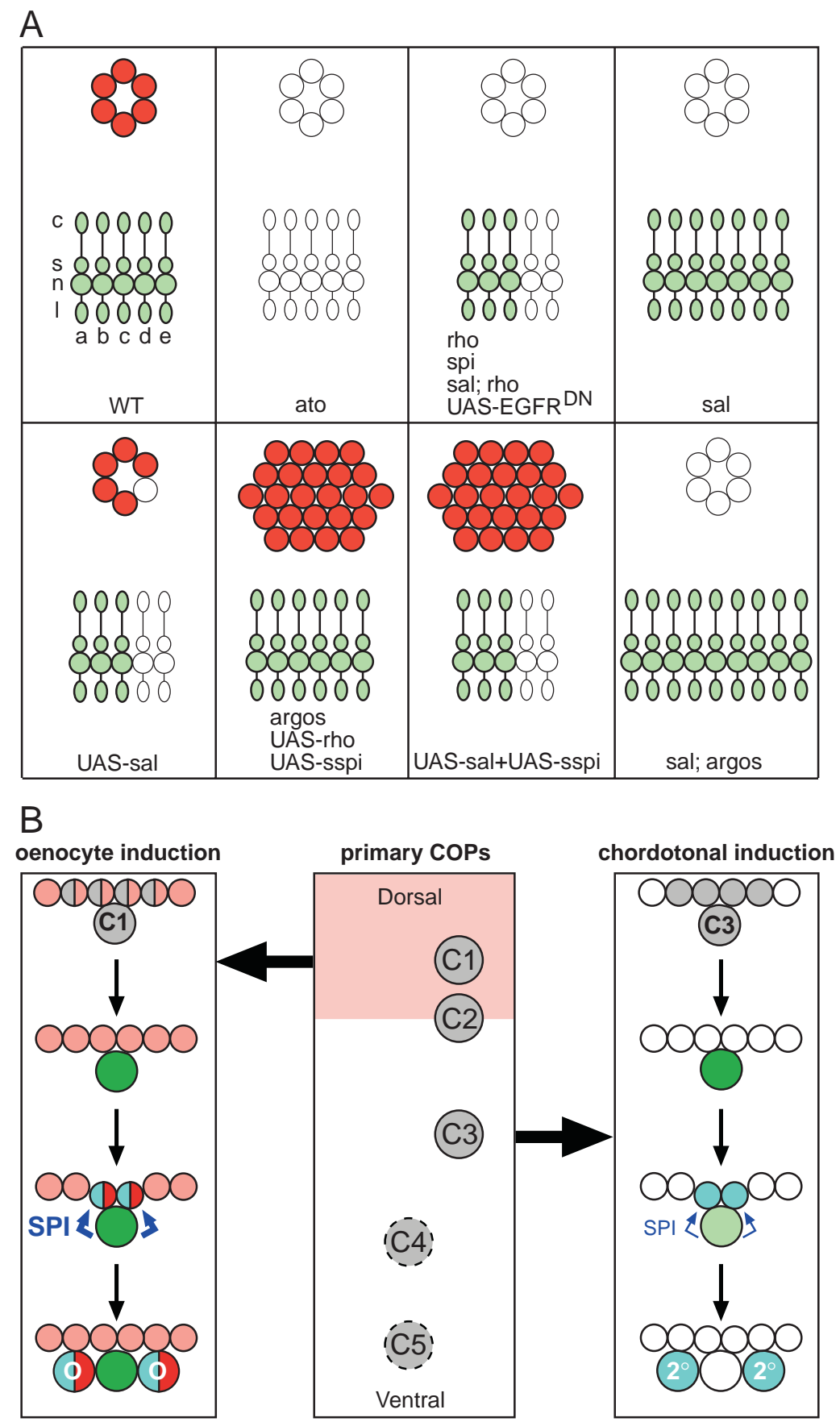

ATO low/high RHO EGFR activation low/high SAL 
explanation for the reduction in precursor number is that increasing the SAL concentration in responding cells reduces their sensitivity to the EGFR ligand produced by $\mathrm{C} 1$. The main conclusion from this experiment, however, is that misexpression of sal in the vicinity of ventrally located COPs is sufficient to suppress chordotonal recruitment but not to promote ectopic oenocyte induction.

To mimic the conditions around $\mathrm{C} 1$ in a wild-type embryo, we tested whether a combination of increased EGFR signaling and the presence of SAL might be sufficient to induce ectopic oenocyte formation in regions outside the dorsal SAL domain. We used the en-GAL4 driver to coexpress sspi and sal (Fig. 5C,D). The effect of this coexpression on the lateral chordotonal organs is to produce Lch3 arrays at high penetrance (Fig. 5D). This represents a suppression of the Lch6-7 forms seen with UAS-sspi and is identical to the phenotype obtained with UAS-sal alone. Hence, with respect to COP induction, overexpression of sal is epistatic to constitutive sspi. This is consistent with the findings in sal; rho and sal; argos double mutants and further supports the conclusion that sal acts downstream or in parallel to SPI ligand production during oenocyte induction and COP repression.

Combined misexpression of sspi and sal does not produce ectopic oenocyte induction in ventral regions. Instead, this results in giant oenocyte clusters (21-35 cells) that are induced dorsally, as seen with excess sspi alone (Fig. 5C and data not shown). Importantly, the reduction in oenocyte numbers seen with sal overexpression can be completely abrogated by simultaneously providing excess SSPI. This strongly suggests that raising the concentration of SAL in oenocyte precursors leads to a reduced sensitivity to induction by SSPI. Such an effect is consistent with the previous results of $\mathrm{EGFR}^{\mathrm{DN}}$ misexpression, where a partial block of receptor activation completely abolished SAL-positive oenocyte induction but not SAL-negative COP recruitment. Such preferential blocking of oenocyte induction by $\mathrm{EGFR}^{\mathrm{DN}}$ can not be explained by reduced SPI ligand production as $\mathrm{C} 1$ expresses $\mathrm{RHO}$ more strongly than the other primary COPs. Taken together, these experiments suggest, but do not prove, that the presence of SAL in the nucleus of the responding cell raises the threshold for induction by SPI.

\section{DISCUSSION}

Little is known about the molecular linkage between the canonical EGFR pathway and the induction of different cell fates. Here we have addressed this issue with a new and relatively simple model system where receipt of SPI signal can only be interpreted in two qualitatively different ways (Fig. 6). We have dissected the mechanism underlying this binary fate switch and find that sal encodes a critical component that functions downstream or in parallel to the EGFR. We discuss the qualitative and quantitative contributions of sal and the EGF pathway and propose a prime-and-respond model that explains how one signaling pathway can trigger the differentiation of two alternative cell fates.

\section{Induction of oenocytes by the PNS}

We have shown that oenocytes are induced from the ectoderm by an inductive signal that is generated in the developing PNS.
The production of active SPI by the $\mathrm{C} 1$ precursor cell, under the control of ato and rho, triggers EGFR activation and thus oenocyte induction in adjacent ectoderm. Oenocyte induction by the PNS appears to be a short-range event with only the cells immediately surrounding $\mathrm{C} 1$ switching on oenocyte markers. In argos mutants, however, the range of the response is increased from one to two concentric rings of cells. Hence, as in photoreceptor recruitment, SPI ligand is not intrinsically limited to immediate neighbors but the response is nevertheless kept short-range by argos-mediated feedback inhibition of the receptor.

In wild-type embryos and in all of the mutant backgrounds that we have examined, the number of cells in the whorl at any one time is less than the final number of mature oenocytes. For example, a wild-type whorl contains 3-4 cells with sickleshaped nuclei but the final oenocyte cluster contains on average 6 cells. We do not yet understand the basis for this difference but note that it might be explained by cell division or by sequential delamination of oenocyte precursors.

\section{Control of cell number by the EGF pathway}

The specification of secondary COP and oenocyte fates requires the EGF pathway. In ato, rho, spi and $E G F R^{D N}$ backgrounds, where signaling is compromised, the induction of both cell types is blocked (Fig. 6A). Conversely, when the EGFR is hyperactivated, both cell types become more numerous. These results indicate that the number of recruited cells is controlled by the amount of EGF pathway signal. It is important to realize, however, that the level, duration and spatial extent of ligand production are all being altered in our experiments. More sophisticated methods would be needed to clearly distinguish which of these three signaling parameters is critical for controlling cell number.

Surprisingly, there is no parity between the numbers of excess oenocytes and lateral chordotonal organs that are produced by EGFR hyperactivation. Thus for a given increase in ligand, more oenocyte precursors than COPs are recruited (Fig. 6A). This implies the existence of an additional tier of control that restricts neural but not oenocyte induction. Such a selective inhibition process would ensure that the number of chordotonal organs is more tightly controlled than that of oenocytes, as is observed in wild-type embryos.

\section{Choice of fate induced by EGF signaling depends on a SAL switch}

As we have described, the expression pattern and mutant phenotype of sal can account for the restriction of oenocyte induction to a single whorl surrounding $\mathrm{C} 1$, the most dorsal primary COP. Previously it was suggested that $\mathrm{C} 1$ and $\mathrm{C} 3$ each induce one secondary COP (zur Lage et al., 1997). However, the results presented here argue that the presence of SAL is incompatible with chordotonal recruitment. Therefore, we favor the idea that $\mathrm{C} 3$ recruits both of the secondary COPs that contribute to the Lch5 array.

The sal gene plays opposite roles in oenocyte and chordotonal induction. It is both necessary and sufficient for repressing secondary COP induction and it is necessary but not sufficient for promoting oenocyte formation. The lack of sufficiency for oenocyte induction is revealed when sal is misexpressed using the en-GAL4 driver. Oenocytes are not ectopically induced in ventral regions, even in the presence of 
excess sspi (Figs 3, 5 and data not shown). It is likely that other factors are required, together with SAL, to promote the oenocyte induction process.

Using epistasis tests and gene expression analysis we have elucidated the regulatory relationship between sal and the EGF pathway in oenocyte and COP formation. These data allow us firmly to exclude the possibility that sal acts upstream of spi in the signaling cell. Importantly, our results indicate that sal functions in the responding ectoderm, either downstream of the EGFR or in a parallel pathway leading to oenocyte induction and secondary COP repression.

In fact, it is probable that sal plays a dual role that is downstream and also in parallel to the EGFR. In rho and spi mutants, the normal upregulation of SAL in the vicinity of $\mathrm{C} 1$ is abolished. Conversely, sspi misexpression produces ectopic SAL upregulation in dorsal locations. Both results indicate that sal lies downstream of the EGFR and that SAL protein levels are controlled by receptor activation. However, SAL is also expressed at moderate levels in presumptive oenocyte precursors prior to EGF pathway activation and this expression remains normal in rho and spi mutants. For these reasons, it is likely that at least part of the function of sal lies in a parallel pathway that, in conjunction with the EGF signal, promotes oenocyte induction and inhibits COP recruitment.

\section{A prime-and-respond model}

To integrate the dual roles of sal downstream and also in parallel to the EGFR, we propose a prime-and-respond model (Fig. 6B). In this model, sal functions in the parallel pathway as a competence switch. Thus, SAL prepatterns the dorsal ectoderm so that, on receipt of the EGF signal, oenocytes rather than COPs are induced. As we have argued, one consequence of the SAL oenocyte prepattern is to increase the apparent induction threshold in responding cells. This makes the prediction that the signaling cell inducing oenocytes needs to express more ligand than those that recruit secondary COPs, and this is indeed the case. $\mathrm{C} 1$ is known to express high levels of rho for longer than any of the other primary COPs (zur Lage et al., 1997). Thus, the EGF pathway does contribute to the cell-type specificity of the induction event in the sense that more SPI ligand is required to induce oenocytes than to recruit chordotonal organs.

One of the early oenocyte-specific responses to the SAL prepattern is the subsequent upregulation of SAL itself. This, in turn, stimulates the expression of the sal target gene $s v p$, one member of the repertoire of oenocyte early differentiation genes (P. E., V. B. and A. G., unpublished). A key feature of the prime-and-respond model is that moderate levels of sal expression serve to prime the responding cells to further upregulate SAL when they receive SPI ligand. In support of this priming mechanism, we have demonstrated that upregulation in response to constitutive sspi expression is restricted to those cells that have already expressed sal. Hence, SAL proteins provide a molecular link between the prepattern and the EGFR response.

In the prime-and-respond model, it is implicit that the early and late phases of sal expression produce distinct effects on the responding cell. As the levels of SAL are different in the two phases, it may be that there are at least two different concentration-dependent effects for this transcription factor. In agreement with this, we have shown that strong expression of the sal target gene, svp, correlates with the domain of sal upregulation and not with the lower-level prepattern. In another system, wing vein development, there is a very extreme example of a concentration difference, with low and high levels of SAL producing completely opposite transcriptional effects on the knirps target gene (de Celis and Barrio, 2000).

\section{PERSPECTIVES}

As the EGF pathway and sal play essential roles in the development of many different tissues, the prime-and-respond mechanism described here may well be deployed in other contexts. For example, in the developing Drosophila tracheal system, it has been suggested that the initial phase of sal expression during primary branch formation is EGFR independent but that later expression in the dorsal trunk is maintained by the EGF pathway (Chen et al., 1998). Furthermore, as has been already stated, there are striking parallels between the oenocyte/chordotonal system and the developing Drosophila eye. In this regard, we note that sal is expressed in the eye imaginal disc in a subset of developing photoreceptor and accessory cells (Barrio et al., 1999). It remains to be seen whether sal or its sister gene, spalt related, also switch the outcome of EGF signaling in this developmental context.

Orthologs of sal have been identified in many animals other than Drosophila. In humans, it has been shown that one sal ortholog is associated with Townes-Brocks syndrome, an autosomal dominant condition affecting numerous structures including the limb (Kohlhase et al., 1998). Moreover, recent studies of the developing chick limb have revealed that a sal ortholog can be regulated by sonic hedgehog, BMP, FGF and Wnt signals (Farrell and Munsterberg, 2000). This raises the possibility that vertebrate sal family genes may be involved in modulating the responses to a wide range of signals and not just EGFR ligands.

We wish to thank R. Schuh, S. Benzer, E. Bier, A. Jarman, M. Freeman, J. de Celis, R. Barrio, F. Kafatos, W. Johnson, J.-P. Vincent, M. Calleja, G. Morata, R. White and the Bloomington and Umea stock centers for stocks and antibodies. We also thank C. Alexandre, B. Bello and S. Pagakis for advice, and J. Smith and J.-P. Vincent for critical reading of the manuscript. This work was supported by the Medical Research Council.

\section{REFERENCES}

Anderson, M. G., Perkins, G. L., Chittick, P., Shrigley, R. J. and Johnson, W. A. (1995). drifter, a Drosophila POU-domain transcription factor, is required for correct differentiation and migration of tracheal cells and midline glia. Genes Dev. 9, 123-137.

Bang, A. G. and Kintner, C. (2000). Rhomboid and Star facilitate presentation and processing of the Drosophila TGF-alpha homolog Spitz. Genes Dev. 14, 177-186.

Barrio, R., de Celis, J. F., Bolshakov, S. and Kafatos, F. C. (1999). Identification of regulatory regions driving the expression of the Drosophila spalt complex at different developmental stages. Dev. Biol. 215, 33-47.

Bier, E., Jan, L. Y. and Jan, Y. N. (1990). rhomboid, a gene required for dorsoventral axis establishment and peripheral nervous system development in Drosophila melanogaster. Genes Dev. 4, 190-203.

Bodenstein, D. (1950). The Postembryonic Development of Drosophila. In Biology of Drosophila (ed. M. Demerec) New York: John Wiley \& Sons.

Boube, M., Llimargas, M. and Casanova, J. (2000). Cross-regulatory 
interactions among tracheal genes support a co-operative model for the induction of tracheal fates in the Drosophila embryo. Mech Dev. 91, 271 278.

Brand, A. H. and Perrimon, N. (1993). Targeted gene expression as a means of altering cell fates and generating dominant phenotypes. Development $\mathbf{1 1 8}$, 401-415.

Brewster, R. and Bodmer, R. (1995). Origin and specification of type II sensory neurons in Drosophila. Development 121, 2923-2936.

Brewster, R. and Bodmer, R. (1996). Cell lineage analysis of the Drosophila peripheral nervous system. Dev. Genet. 18, 50-63.

Chen, C. K., Kuhnlein, R. P., Eulenberg, K. G., Vincent, S., Affolter, M. and Schuh, R. (1998). The transcription factors KNIRPS and KNIRPS RELATED control cell migration and branch morphogenesis during Drosophila tracheal development. Development 125, 4959-4968.

de Celis, J. F. and Barrio, R. (2000). Function of the spalt/spalt-related gene complex in positioning the veins in the Drosophila wing. Mech. Dev. 91, 31-41.

de Celis, J. F., Bray, S. and Garcia-Bellido, A. (1997). Notch signalling regulates veinlet expression and establishes boundaries between veins and interveins in the Drosophila wing. Development 124, 1919-1928.

Dickson, B., Sprenger, F. and Hafen, E. (1992). Prepattern in the developing Drosophila eye revealed by an activated torso-sevenless chimeric receptor. Genes Dev. 6, 2327-2339.

Farrell, E. R. and Munsterberg, A. E. (2000). csal1 is controlled by a combination of FGF and Wnt signals in developing limb buds. Dev. Biol. 225, 447-458.

Flores, G. V., Duan, H., Yan, H., Nagaraj, R., Fu, W., Zou, Y., Noll, M. and Banerjee, U. (2000). Combinatorial signaling in the specification of unique cell fates. Cell 103, 75-85.

FlyBase. (1999). The FlyBase database of the Drosophila Genome Projects and community literature. The FlyBase Consortium. Nucleic Acids Res. 27, 85-88.

Freeman, M. (1994). The spitz gene is required for photoreceptor determination in the Drosophila eye where it interacts with the EGF receptor. Mech. Dev. 48, 25-33.

Freeman, M. (1996). Reiterative use of the EGF receptor triggers differentiation of all cell types in the Drosophila eye. Cell 87, 651-660.

Freeman, M., Kimmel, B. E. and Rubin, G. M. (1992a). Identifying targets of the rough homeobox gene of Drosophila: evidence that rhomboid functions in eye development. Development 116, 335-346.

Freeman, M., Klambt, C., Goodman, C. S. and Rubin, G. M. (1992b). The argos gene encodes a diffusible factor that regulates cell fate decisions in the Drosophila eye. Cell 69, 963-975.

Fujita, S. C., Zipursky, S. L., Benzer, S., Ferrus, A. and Shotwell, S. L. (1982). Monoclonal antibodies against the Drosophila nervous system. Proc. Natl. Acad. Sci. USA 79, 7929-7933.

Golembo, M., Schweitzer, R., Freeman, M. and Shilo, B. Z. (1996). Argos transcription is induced by the Drosophila EGF receptor pathway to form an inhibitory feedback loop. Development 122, 223-230.

Halfon, M. S., Carmena, A., Gisselbrecht, S., Sackerson, C. M., Jimenez, F., Baylies, M. K. and Michelson, A. M. (2000). Ras pathway specificity is determined by the integration of multiple signal-activated and tissuerestricted transcription factors. Cell 103, 63-74.

Hartenstein, A. Y., Rugendorff, A., Tepass, U. and Hartenstein, V. (1992). The function of the neurogenic genes during epithelial development in the Drosophila embryo. Development 116, 1203-1220.

Hummel, T., Krukkert, K., Roos, J., Davis, G. and Klambt, C. (2000). Drosophila Futsch/22C10 is a MAP1B-like protein required for dendritic and axonal development. Neuron 26, 357-370.

Jarman, A. P., Grau, Y., Jan, L. Y. and Jan, Y. N. (1993). atonal is a proneural gene that directs chordotonal organ formation in the Drosophila peripheral nervous system. Cell 73, 1307-1321.

Jarman, A. P., Grell, E. H., Ackerman, L., Jan, L. Y. and Jan, Y. N. (1994). Atonal is the proneural gene for Drosophila photoreceptors. Nature $\mathbf{3 6 9}$, 398-400.

Jarman, A. P., Sun, Y., Jan, L. Y. and Jan, Y. N. (1995). Role of the proneural gene, atonal, in formation of Drosophila chordotonal organs and photoreceptors. Development 121, 2019-2030.

Jurgens, G. (1988). Head and tail development in the Drosophila embryo involves spalt, a novel homeotic gene. EMBO J. 7, 189-196.
Jurgens, G., Wieschaus, E., Nusslein-Volhard, C. and Kluding, H. (1984) Mutations affecting the pattern of the larval cuticle in Drosophila melanogaster II. Zygotic loci on the third chromosome. Roux's Arch. Dev. Biol. 193, 283-295.

Kohlhase, J., Wischermann, A., Reichenbach, H., Froster, U. and Engel, W. (1998). Mutations in the SALL1 putative transcription factor gene cause Townes-Brocks syndrome. Nat. Genet. 18, 81-83.

Kuhnlein, R. P., Frommer, G., Friedrich, M., Gonzalez-Gaitan, M., Weber, A., Wagner-Bernholz, J. F., Gehring, W. J., Jackle, H. and Schuh, R. (1994). spalt encodes an evolutionarily conserved zinc finger protein of novel structure which provides homeotic gene function in the head and tail region of the Drosophila embryo. EMBO J. 13, 168-179.

Kuhnlein, R. P. and Schuh, R. (1996). Dual function of the region-specific homeotic gene spalt during Drosophila tracheal system development. Development 122, 2215-2223.

Mlodzik, M., Hiromi, Y., Weber, U., Goodman, C. S. and Rubin, G. M. (1990). The Drosophila seven-up gene, a member of the steroid receptor gene superfamily, controls photoreceptor cell fates. Cell 60, 211-224.

Nusslein-Volhard, C., Wieschaus, E. and Kluding, H. (1984). Mutations affecting the pattern of the larval cuticle in Drosophila melanogaster I. Zygotic loci on the second chromosome. Roux's Arch. Dev. Biol. 193, 267282.

O'Keefe, L., Dougan, S. T., Gabay, L., Raz, E., Shilo, B. Z. and DiNardo, S. (1997). Spitz and Wingless, emanating from distinct borders, cooperate to establish cell fate across the Engrailed domain in the Drosophila epidermis. Development 124, 4837-4845.

O’Neill, E. M., Rebay, I., Tjian, R. and Rubin, G. M. (1994). The activities of two Ets-related transcription factors required for Drosophila eye development are modulated by the Ras/MAPK pathway. Cell 78, 137-147.

Okabe, M. and Okano, H. (1997). Two-step induction of chordotonal organ precursors in Drosophila embryogenesis. Development 124, 1045-1053.

Okabe, M., Sawamoto, K. and Okano, H. (1996). The function of the Drosophila argos gene product in the development of embryonic chordotonal organs. Dev. Biol. 175, 37-49.

Perrimon, N. and Perkins, L. A. (1997). There must be 50 ways to rule the signal: the case of the Drosophila EGF receptor. Cell 89, 13-16.

Rutledge, B. J., Zhang, K., Bier, E., Jan, Y. N. and Perrimon, N. (1992). The Drosophila spitz gene encodes a putative EGF-like growth factor involved in dorsal-ventral axis formation and neurogenesis. Genes Dev. 6, 1503-1517.

Scholz, H., Deatrick, J., Klaes, A. and Klambt, C. (1993). Genetic dissection of pointed, a Drosophila gene encoding two ETS- related proteins. Genetics 135, 455-468.

Schweitzer, R., Shaharabany, M., Seger, R. and Shilo, B. Z. (1995). Secreted Spitz triggers the DER signaling pathway and is a limiting component in embryonic ventral ectoderm determination. Genes Dev. 9, 1518-1529.

Schweitzer, R. and Shilo, B. Z. (1997). A thousand and one roles for the Drosophila EGF receptor. Trends Genet. 13, 191-196.

Simon, M. A. (2000). Receptor tyrosine kinases: specific outcomes from general signals. Cell 103, 13-15.

Sturtevant, M. A., Roark, M., O’Neill, J. W., Biehs, B., Colley, N. and Bier, E. (1996). The Drosophila rhomboid protein is concentrated in patches at the apical cell surface. Dev. Biol. 174, 298-309.

Tio, M., Ma, C. and Moses, K. (1994). spitz, a Drosophila homolog of transforming growth factor-alpha, is required in the founding photoreceptor cells of the compound eye facets. Mech. Dev. 48, 13-23.

Wappner, P., Gabay, L. and Shilo, B. Z. (1997). Interactions between the EGF receptor and DPP pathways establish distinct cell fates in the tracheal placodes. Development 124, 4707-4716.

Wasserman, J. D., Urban, S. and Freeman, M. (2000). A family of rhomboid-like genes: Drosophila rhomboid-1 and roughoid/rhomboid-3 cooperate to activate EGF receptor signaling. Genes Dev. 14, 1651-1663.

Xu, C., Kauffmann, R. C., Zhang, J., Kladny, S. and Carthew, R. W. (2000). Overlapping activators and repressors delimit transcriptional response to receptor tyrosine kinase signals in the Drosophila eye. Cell $\mathbf{1 0 3}$ 87-97.

zur Lage, P., Jan, Y. N. and Jarman, A. P. (1997). Requirement for EGF receptor signalling in neural recruitment during formation of Drosophila chordotonal sense organ clusters. Curr. Biol. 7, 166-175. 